\title{
AN EXISTENCE THEOREM FOR DIFFERENTIAL INCLUSIONS ON BANACH SPACE ${ }^{1}$
}

\author{
N.U. AHMED \\ University of Ottawa \\ Department of Mathematics and \\ Department of Electrical Engineering \\ Ottawa, CANADA
}

\begin{abstract}
In this paper we consider the question of existence of solutions for a large class of nonlinear differential inclusions on Banach space arising from control theory.
\end{abstract}

Key words: Existence, differential inclusions, Banach space, Sousline space, multifunctions, uncertain systems, optimal control.

AMS (MOS) subject classifications: $34 \mathrm{Gxx}, 34 \mathrm{G} 20,35 \mathrm{~A} 05$, 47H04, 49J24, 93C25.

\section{INTRODUCTION AND MOTIVATION}

We consider the system governed by a differential inclusion given by

$$
\begin{aligned}
(d / d t) x(t)+A(t, x(t)) & \in B(t) u(t)+G(t, x(t)) \\
x(0) & =x_{0},
\end{aligned}
$$

where $G(t, x)$ is a suitable set valued map or multi function. For example, $G$ may be given by $G(t, x)=\left\{y: y=\int_{\Sigma} g(t, x, \alpha) \mu(d \alpha), \mu \in \mathcal{H}^{+}(\Sigma)\right\}$ where $g$ is a suitable function and $\mathcal{H}^{+}(\Sigma)$ is the space of probability measures on the Borel fields $\mathfrak{B}(\Sigma)$ of (possibly) a Souslin space $\Sigma$. In the case of systems with parametric or structural uncertainties the parameter $\alpha$ or even its probability law is usually unknown and in that situation the differential inclusion model (1) is preferable to the evolution equation model obtained by replacing the set map $G$ by any of its selections $g$ which are only vaguely known $[3,6]$. The logical control problem here would be to minimize the maximum risk. Another motivation comes from the fact that systems governed by evolution inequalities of the form,

$$
\langle(d / d t) x+A(t, x(t))-B(t) u(t), x(t)-w\rangle \geq g(t, x(t))-g(t, w) \text { for all } w \in V
$$

\footnotetext{
${ }^{1}$ Received: June, 1991. Revised: October, 1991.
} 


$$
x(0)=x_{0}
$$

can be described by a differential inclusion. For example, suppose for each $t \geq 0$, and each $\xi \in V$, the function $\eta \rightarrow g(t, \eta)$ from $V$ to $R$ has the sub differential $\partial g(t, \xi) \equiv G(t, \xi)$. Then one can easily verify that the above evolution inequality is equivalent to the differential inclusion (1). In case $\xi \rightarrow g(t, \xi)$ is convex, $G(t, \xi)$ is, in general, a multivalued monotone operator from $\boldsymbol{V}$ to $V^{*}$. However if $g$ is Gateaux differentiable, then $G(t, \cdot)$ is a single valued operator from $V$ to $V^{*}$. If in $\left(1^{\prime}\right) \boldsymbol{V}$ is replaced by a nonempty closed convex subset $K \subseteq \boldsymbol{V}$ then we have an obstacle problem. Thus the differential inclusion model (1) can be considered to be an abstract model for many physical problems.

Here we are interested in the basic question of existence and regularity properties of solutions of the general differential inclusion (1) on a Banach space. We combine a selection theorem in Banach space, Galerkin's approximation, an existence result for differential inclusions on finite dimensional spaces, and compactness arguments to prove our main result. This is in contrast to Kakutani-Fan fixed point theorem found useful for semilinear inclusions [3].

\section{BASIC NOTATIONS AND ASSUMPTIONS}

Let $H$ be a separable Hilbert space and $V$ a subspace of $H$ having the structure of a reflexive Banach space with the embedding $V \rightarrow H$ being continuous and dense. Identifying $H$ with its dual we have $V \rightarrow H \rightarrow V^{*}$ where $V^{*}$ is the topological dual of $V$. Let $\langle y, x\rangle$ denote the pairing of an element $x \in V$ with an element $y \in V^{*}$. If $x, y \in H$, then $\langle y, x\rangle=(y, x)$ where $($, denotes the scalar product in $H$. The norm in any Banach space $X$ will be denoted by $\|\cdot\|_{X}$. We shall use $\mathscr{P}(\boldsymbol{X})(c c(\boldsymbol{X}), c b c(\boldsymbol{X}))$ to denote the class of all nonempty (nonempty closed convex, nonempty closed bounded convex) subsets of $\boldsymbol{X}$.

Let $I \equiv[0, T], 0<T<\infty$, and $p, q \geq 1$ such that $(1 / p)+(1 / q)=1$ and $2 \leq p<\infty$. Denote $L_{p}(V) \equiv L_{p}(I, V), L_{q}\left(V^{*}\right) \equiv L_{q}\left(I, V^{*}\right)$. For $p, q$ satisfying the preceding conditions, it follows from the reflexivity of $V$ that both $L_{p}(V)$ and $L_{q}\left(V^{*}\right)$ are reflexive Banach spaces. The pairing between $L_{p}(V)$ and $L_{q}\left(V^{*}\right)$ is denoted by $《\left(\eta\right.$. Clearly, for $u, v \in L_{2}(H)$, $\langle\langle u, v\rangle\rangle=((u, v))$ is the scalar product in the Hilbert space $L_{2}(H)$. Our results are based on the following basic assumptions:

(A) $\quad A: I \times V \rightarrow V^{*}$ is a map so that

(1): $\quad t \rightarrow A(t, x)$ is measurable,

(2): $\quad x \rightarrow A(t, x)$ is monotone and hemicontinuous, that is, $\langle A(t, x)-A(t, y), x-y\rangle \geq 0$ and $A(t, x+\sigma y) \stackrel{w}{\rightarrow} A(t, x)$ in $V^{*}$ as $\sigma \rightarrow 0$ for all $x, y \in V, \quad t \in I ;$ and 


$$
\left\langle\left\langle A\left(\zeta_{n}\right)-A(\xi), \zeta_{n}-\xi\right\rangle\right\rangle \rightarrow 0 \text { as } \zeta_{n} \rightarrow \xi \text { weakly in } L_{p}(V) \text {. }
$$

(3): there exist positive constants $c_{1}, c_{2}, c_{3}$ such that

$\|A(t, x)\|_{V^{*}} \leq c_{1}\left(1+\|x\| P^{-1}\right)$ and $\langle A(t, x), x\rangle+c_{3} \geq c_{2}\|x\| p$ for all $x \in V$.

$(G): \quad G: I \times V \rightarrow c c(H)$ satisfying the following properties:

(1) $t \rightarrow G(t, x)$ is a measurable multifunction.

(2) $(t, x) \rightarrow G(t, x)$ is sequentially upper hemicontinuous with respect to inclusion and further, the multifunction $\hat{G}$, defined by

$$
x \rightarrow \hat{G}(x)=\left\{\xi \in L_{q}(H): \xi(t) \in G(t, x(t)) \text { a.e. }\right\}, \quad \text { is sequentially weakly upper }
$$

semicontinuous with respect to inclusion from $L_{p}(V)$ to $\mathscr{P}\left(L_{q}(H)\right)$ whenever it is nonempty.

(3) there exist positive numbers $\alpha, \beta, \gamma$ such that for all $\{t, x\} \in I \times V(y, x) \leq \gamma$, and $\|y\|_{H} \leq \alpha\|x\| p^{-1}+\beta$ for each $y \in G(t, x)$.

(B): $\quad B \in L_{\infty}(I, \mathcal{L}(Y, H))$ with $Y$ being a reflexive Banach space where controls take their values from.

$(U): \quad U: I \rightarrow c c(Y)$ is a measurable multifunction satisfying $U(t) \subseteq \mathcal{U}$ for almost all $t \in I$ where $\mathcal{U}$ is a fixed weakly compact convex subset of $Y$. For the admissible controls we choose the set

$$
\mathcal{u}_{a d} \equiv\left\{u \in L_{\infty}(Y): u(t) \in U(t) \text { a.e. }\right\} \text {. }
$$

\section{MAIN RESULT}

For convenience of notation we shall use $D x$ to denote $(d / d t) x$. For $p, q$ satisfying the conditions stated above define

$$
W_{p, q} \equiv\left\{x \in L_{p}(V): D x \equiv(d / d t) x \in L_{q}\left(V^{*}\right)\right\}
$$

where the derivative is understood in the sense of distributions. Furnished with the norm topology $\|x\|_{p, q}$, defined by $\|x\|_{p, q}^{2} \equiv\left(\|x\|_{L_{p}(V)}^{2}+\|D x\|_{L_{q}\left(V^{*}\right)}^{2}\right), W_{p, q}$ is a Banach space and it follows from theorem 1.1.7 of [1], that the embedding $W_{p, q} \rightarrow C(I, H)$ is continuous.

Definition 1: A function $x \in C(I, H)$ is said to be a solution of the differential inclusion (1) if there exists a measurable selection $y$ so that $y(t) \in G(t, x(t))$ a.e. and $x$ satisfies, in the sense of $V^{*}$-valued distributions, the evolution equation

$$
D x(t)+A(t, x(t))=B(t) u(t)+y(t) \text { for almost all } t \in I \text {. }
$$

Our main existence result is given in the following theorem. 
Theorem 2: Consider the system (1) and suppose the assumptions $(A),(G),(B)$ and $(U)$ hold. Then for every $x_{0} \in H$ and $u \in \mathcal{U}_{a d}$ the system (1) has at least one solution $x \in W_{p, q}$.

Proof: By using Galerkin approach, we reduce this problem to the problem of existence of solutions of a differential inclusion on a finite dimensional space and then use weak compactness along with limiting arguments and upper semicontinuity of $G$ to complete the proof. For this we first establish an a priori bound for the solutions of (1). Let $x \in W_{p, q}$ be any solution of $(1)$ and define $\mathrm{g}(t) \equiv G(t, x(t))$ and $y$ a measurable selection of $g$. By virtue of the growth assumption [see assumption $(G)$ ] it follows that $y \in L_{q}(H)$. Scalar multiplying (2) by $x$ and integrating over $[0, t]$ we have

$$
\begin{gathered}
\|x(t)\|_{H}^{2}+2 \int_{0}^{t}\langle A(s, x(s)), x(s)\rangle d s \\
=\left\|x_{0}\right\|_{H}^{2}+2 \int_{0}^{t}\{\langle B(s) u(s), x(s)\rangle+\langle y(s), x(s)\rangle\} d s .
\end{gathered}
$$

Letting $\delta$ denote the embedding constant $V \rightarrow H, M \equiv \max \left\{\|v\|_{\boldsymbol{Y}}, v \in \mathcal{U}\right\}$ and using the assumptions $(A 3),(G 3),(U)$ and Cauchy inequality one can easily verify that

$$
\begin{gathered}
\|x(t)\|_{H}^{2}+2 c_{2} \int_{0}^{t}\|x(s)\| V^{p} d s \leq T\left(2 c_{3}+\gamma\right)+\left\|x_{0}\right\|_{H}^{2}+\left(1 / q \epsilon^{q}\right)\|B\|_{L_{q}(\mathcal{L}(Y, H))}^{q} \\
+\left((2 \delta M \epsilon)^{p} / p\right) \int_{0}^{t}\|x(s)\|{ }^{p} d s
\end{gathered}
$$

for every $\epsilon>0$. Choosing $\epsilon \equiv\left(\left(c_{2} p\right)^{(1 / p)} / 2 \delta M\right)$ it follows from this expression that there exists a constant $c_{4}$ dependent only on the parameters $T, \gamma, c_{2}, c_{3}, \beta, M, p, q,\left\|x_{0}\right\|_{H}$ and $\|B\|_{L_{q}(\mathcal{L}(Y, H))}$ so that

$$
\|x(t)\|_{H}^{2}+c_{2} \int_{0}^{t}\|x(s)\| P^{p} d s \leq c_{4} \text { for all } t \in I \equiv[0, T]
$$

This proves that $x \in L_{\infty}(H) \cap L_{p}(V)$ and that the bound (4) holds for all solutions of (1). Scalar multiplying $(2)$ by $\eta \in L_{p}(V)$ one can easily verify using the assumptions $(A 3),(G 3)$, $(B),(U)$ and $(4)$ that there exists a constant $c_{5}$ dependent only on the parameters $c_{1}, c_{4}, T, \alpha$, $\beta, \delta, M, p, q$ and $\|B\|_{L_{q}(\mathcal{L}(Y, H))}$ such that

$$
|\langle\langle D x, \eta\rangle\rangle|=\left|\int_{I}\langle D x(s), \eta(s)\rangle d s\right| \leq c_{5}\|\eta\|_{L_{p}(V)} .
$$

This shows that $D x \in L_{q}\left(V^{*}\right)$ and that $\|D x\|_{L_{q}\left(V^{*}\right)} \leq c_{5}$ for all $x$ that satisfy (4). Thus, for 
$c_{6} \equiv \operatorname{Max}\left\{c_{4}, c_{5}\right\}$, all solutions of (1) satisfy

$$
\|x\|_{L_{\infty}(H)} \leq c_{6},\|x\|_{L_{p}(V)} \leq c_{6},\|D x\|_{L_{q}\left(V^{*}\right)} \leq c_{6} .
$$

Clearly this shows that all the solutions of (1) lie in a bounded subset of the Banach space $W_{p, q}$. With this a priori bound, we are now ready to prove the existence of solutions. Since $V$ is a separable Banach space and the injection $V \rightarrow H$ is dense there exists $\left\{e_{i}\right\} \subseteq V$ so that $\left(e_{i}, e_{j}\right)_{H}=\delta_{i j}$ which forms a basis for $V, H$ and also $V^{*}$. Since $x_{0} \in H$ we have

$$
x_{0}=s-\lim \sum_{1 \leq i \leq n} \eta_{i} e_{i} \text { where } \eta_{i} \equiv\left(x_{0}, e_{i}\right)
$$

Define

$$
x_{n}(t) \equiv \sum_{1 \leq i \leq n} \xi_{i}^{n}(t) e_{i}
$$

and choose $\xi^{n} \equiv\left(\xi_{i}^{n}, 1 \leq i \leq n\right)$ in such a way that $\xi_{i}^{n}(0)=\eta_{i}, 1 \leq i \leq n$ and

$$
\begin{gathered}
\left\langle D x_{n}(t), e_{k}\right\rangle+\left\langle A\left(t, x_{n}(t)\right), e_{k}\right\rangle=\left(B(t) u(t), e_{k}\right)+\left(y_{n}(t), e_{k}\right), \\
\text { and } y_{n}(t) \in G_{n}(t) \equiv G\left(t, x_{n}(t)\right), \text { for } t \in I, 1 \leq k \leq n
\end{gathered}
$$

where $y_{n}$ is a measurable selection of the set valued map $G_{n}(\cdot) \equiv G\left(\cdot x_{n}(\cdot)\right)$. The existence of a measurable selection is justified later. First we verify the existence of $\xi^{n}$. For each $(t, \zeta) \in I \times R^{n}$ define

$$
\begin{gathered}
F(t, \zeta) \equiv\left\{z \in R^{n}: z_{k}=-\left\langle A\left(t, \sum_{1 \leq i \leq n} \zeta_{i} e_{i}\right), e_{k}\right\rangle+\left(B(t) u(t), e_{k}\right)+\left(\vartheta, e_{k}\right)\right. \\
\text { for } \left.\vartheta \in G\left(t, \sum_{1 \leq i \leq n} \zeta_{i} e_{i}\right), 1 \leq k \leq n\right\} .
\end{gathered}
$$

Clearly $F: I \times R^{n} \rightarrow \mathscr{P}\left(R^{n}\right)$. Given that a measurable selection exists, for each $n \in N$, the system (9) is equivalent to the differential inclusion in $R^{n}$ given by

$$
D \xi(t) \in F(t, \xi(t)), t \in I, \xi(0)=\eta^{n} \equiv\left(\eta_{i}, 1 \leq i \leq n\right) .
$$

It follows from assumption $(G)$ that, for $(t, x) \in I \times V, G(t, x) \in c b c(H)$ and hence weakly compact. Therefore, under the assumptions $(B)$ and $(U)$, for a fixed $u \in \mathcal{U}_{a d}$, $F: I \times R^{n} \rightarrow c b c\left(R^{n}\right)$ with measurability in $t$ and upper semicontinuity in $\zeta$ following from the measurability and upper hemicontinuity of $G$. Thus it follows from theorem 2.1.4 [5] that (11) has at least one solution $\xi \in A C\left(I, R^{n}\right)$. Using this $\xi$ in (8) we conclude that $x_{n}$ is a solution of the system (9). It follows from the a priori estimate (6) that $\left\|x_{n}\right\|_{L_{p}(V)} \leq c_{6}$ and $\left\|D x_{n}\right\|_{L_{q}\left(V^{*}\right)} \leq c_{6}$ and, by virtue of the growth assumption $(G 3)$ for $G$, there exists a constant 
$c_{7}$ dependent only on $\alpha, \beta, p, T$, and $c_{6}$ such that $\left\|y_{n}\right\|_{L_{q}(H)} \leq c_{7}$ for all $n \geq 1$. Since the spaces $L_{p}(V), L_{q}\left(V^{*}\right)$ and $L_{q}(H)$ are all reflexive there exists a subsequence of the sequence $\left\{x_{n}, D x_{n}, y_{n}\right\}$, relabeled as such, and $x^{0} \in W_{p, q}$ and $y^{0} \in L_{q}(H)$ such that as $n \rightarrow \infty$

$$
\begin{gathered}
x_{n} \stackrel{w}{\rightarrow} x^{0} \text { in } L_{p}(V) \\
D x_{n} \stackrel{w}{\rightarrow} D x^{0} \text { in } L_{q}\left(V^{*}\right) \\
y_{n} \stackrel{w}{\rightarrow} y^{0} \text { in } L_{q}(H) .
\end{gathered}
$$

Let $A(x)$ denote the function $A(t, x(t)), t \in I$. Under the assumptions $(A)$ it follows from Lemma 3.3 [2] that $A\left(x_{n}\right)^{\stackrel{w}{\rightarrow}} A\left(x^{0}\right)$ in $L_{q}\left(V^{*}\right)$ whenever $x^{n} \stackrel{w}{\rightarrow} x^{0}$ in $L_{p}(V)$. Hence, multiplying the first equation of $(9)$ by a $C^{\infty}(I)$ function $\psi$ with compact support and integrating over $I$ and letting $n \rightarrow \infty$, we obtain

$$
\begin{aligned}
& \int_{I}\left\langle D x^{0}, e_{k}\right\rangle \psi(s) d s+\int_{I}\left\langle A\left(s, x^{0}(s)\right), e_{k}\right\rangle \psi(s) d s \\
= & \int_{I}\left(B(s) u(s), e_{k}\right) \psi(s) d s+\int_{I}\left(y^{0}(s), e_{k}\right) \psi(s) d s .
\end{aligned}
$$

Since $\left\{e_{i}\right\}$ is a basis for $V$ and $\psi \in C_{0}^{\infty}(I)$ is arbitrary, it follows from (13) that, for $y=y^{0}, x^{0}$ satisfies the evolution equation (2) in the sense of $V^{*}$-valued distributions. One can easily verify that $x^{0}(0)=x_{0}$. It remains to show that $y^{0}(t) \in G\left(t, x^{0}(t)\right)$ a.e. It suffices to show that $y^{0} \in \hat{G}\left(x^{0}\right)$ given that $\hat{G}$ is nonempty. For every $x \in L_{p}(V)$, it follows from assumption (G3) that $\hat{G}(x)$ is a bounded subset of $L_{q}(H)$. Since $G(t, \zeta) \in c c(H)$, by use of Hahn-Banach theorem, one can easily verify that $\hat{G}(x)$ is a (strongly) closed convex subset of $L_{q}(H)$. Therefore by Mazur's theorem it is also weakly closed. Hence for every $x \in L_{p}(V), \hat{G}(x)$ is a weakly closed bounded convex subset of $L_{q}(H)$, that is, $\hat{G}$ maps $L_{p}(V)$ to $c b c\left(L_{q}(H)\right)$. Let $\hat{G}_{\epsilon}\left(x^{0}\right)$ denote the closed $\epsilon$-neighborhood of $\hat{G}\left(x^{0}\right)$ in $L_{q}(H)$. Then, by virtue of assumption $(G 2)$, for every $\epsilon>0$ there exists $n_{0} \equiv n_{0}(\epsilon)$ such that $\hat{G}\left(x_{n}\right) \subseteq \hat{G}_{\epsilon}\left(x^{0}\right)$ for all $n \geq n_{0}$. Thus $y_{n} \in \hat{G}\left(x_{n}\right) \subseteq \hat{G}_{\epsilon}\left(x_{0}\right)$ for $n \geq n_{0}$. Since $y^{0}$ is the weak limit of $y_{n}$ in $L_{q}(H)$ and $\hat{G}_{\epsilon}\left(x^{0}\right)$ is a weakly closed convex set, again, by Hahn-Banach theorem, $y^{0} \in \hat{G}_{\epsilon}\left(x^{0}\right)$. But $\epsilon>0$ is arbitrary and $\hat{G}\left(x^{0}\right) \in c b c\left(L_{q}(H)\right)$ and hence $y^{0} \in \hat{G}\left(x^{0}\right)$. Thus we have proved that $y^{0}(t) \in G\left(t, x^{0}(t)\right)$ for almost all $t \in I$. We conclude the proof by justifying the existence of a measurable selection $y_{n}$ for the multifunction $G_{n}$ as required by the inclusion in (9). This will also justify that $\hat{G}$ is nonempty. Since $x_{n} \in A C(I, V)$ it follows from the first part of assumption $(G 2)$ that $t \rightarrow G_{n}(t)$ is upper semicontinuous and hence for every sequence $t_{i} \rightarrow t_{0}$, we have

$$
\bigcap_{k \geq 1} c l \bigcup_{i \geq k} G_{n}\left(t_{i}\right) \subseteq G_{n}\left(t_{0}\right)
$$


Further, it follows from the growth assumption $(G 3)$ that, for each $t \in I, G_{n}(t) \in c b c(H)$ and hence weakly compact. Therefore by the selection theorem $5.4 .3[1$, p. 378], it has a measurable selection which we have denoted by $y_{n}$. This completes the proof.

Corollary 3: For $u \in \mathcal{U}_{a d}$, the set $\mathscr{S}(u)$, denoting the family of solutions of (1) corresponding to the control $u$ and the initial state $x_{0}$, is a bounded weakly closed subset of $W_{p, q}$ and hence weakly compact.

Remark 4: As far as the existence question is concerned, Theorem 2 also holds under the relaxed conditions: $B \in L_{\infty}\left(\mathcal{L}\left(Y, V^{*}\right)\right), G: I \times V \rightarrow \mathscr{P}\left(V^{*}\right)$ satisfying the assumption $(G)$ with $H$ replaced by $V^{*}$ and $\beta \in L_{q}(I, R)$. For control problems, however, we need the stronger assumptions.

If the initial data is assumed to belong to $V$, the assumption $(G 3)$ may be relaxed.

\section{ACKNOWLEDGEMENT}

The author wishes to thank the anonymous reviewer for pointing out an error in our original proof.

\section{REFERENCES}

[1] N.U. Ahmed and K.L. Teo, "Optimal Control of Distributed Parameter Systems", North Holland, New York, Oxford, 1981.

[2] N.U. Ahmed, "Optimal control of a class of strongly nonlinear parabolic systems", JMAA 61, 1, (1977), pp. 188-207.

[3] N.U. Ahmed, "Existence of optimal controls for a class of systems governed by differential inclusions on a Banach space", JOTA 50, 2, (1986), pp. 213-237.

[4] N.U. Ahmed, "Optimization and identification of systems governed by evolution equations on Banach space", Pitman Res. Notes in Math. Ser., Vol. 184, Longman Scientific \& Technical England \& John Wiley \& Sons, New York, 1988.

[5] J.P. Aubin and A. Cellina, "Differential Inclusions", Springer-Verlag, Berlin, Heidelberg, New York, Tokyo, 1984.

[6] N.S. Papageorgiou, "Optimal control of nonlinear evolution equations", Publicationes Matehematicae Debrecen, Hungary, (to appear). 


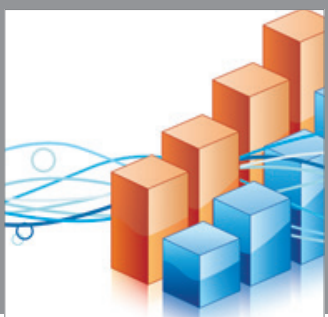

Advances in

Operations Research

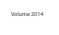

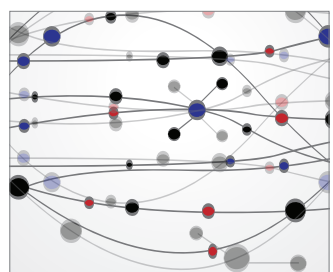

\section{The Scientific} World Journal
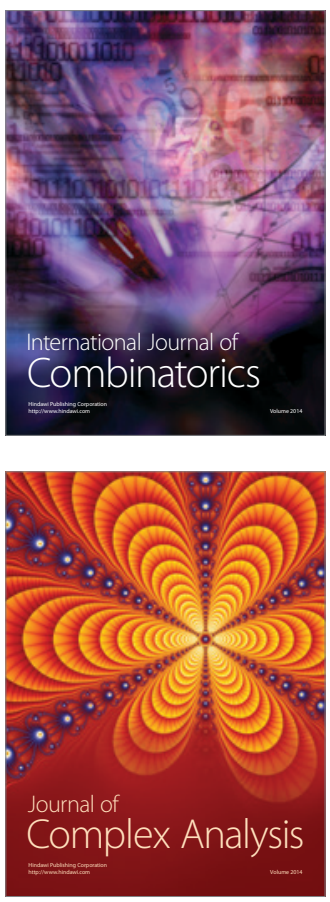

International Journal of

Mathematics and

Mathematical

Sciences
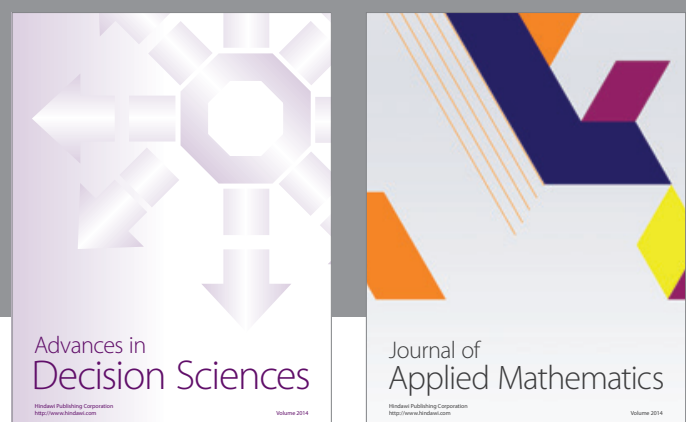

Journal of

Applied Mathematics
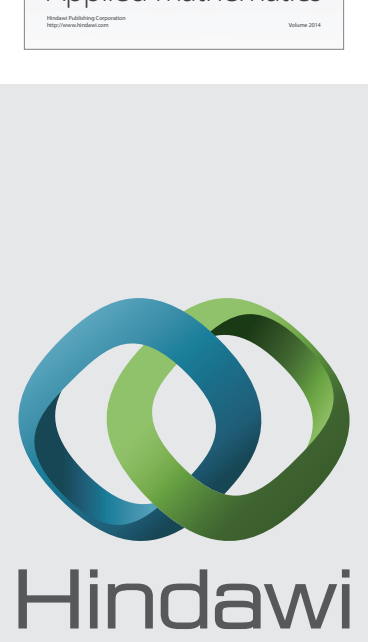

Submit your manuscripts at http://www.hindawi.com
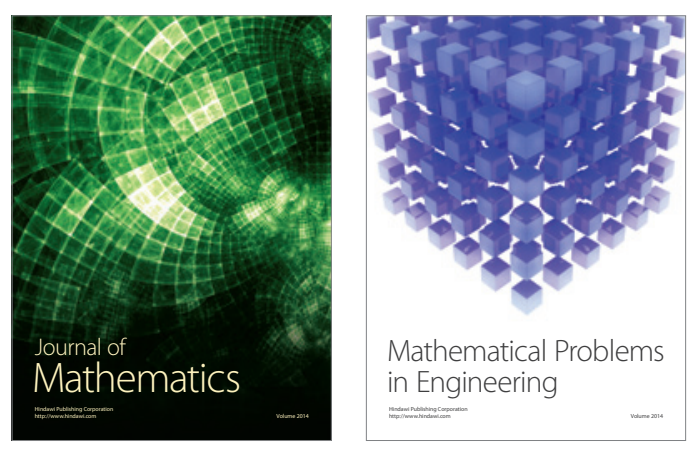

Mathematical Problems in Engineering
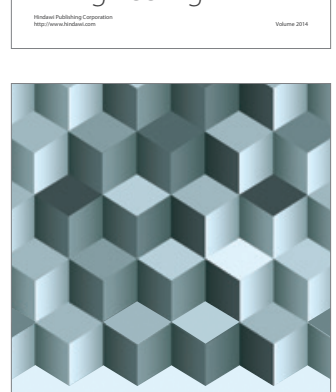

Journal of

Function Spaces
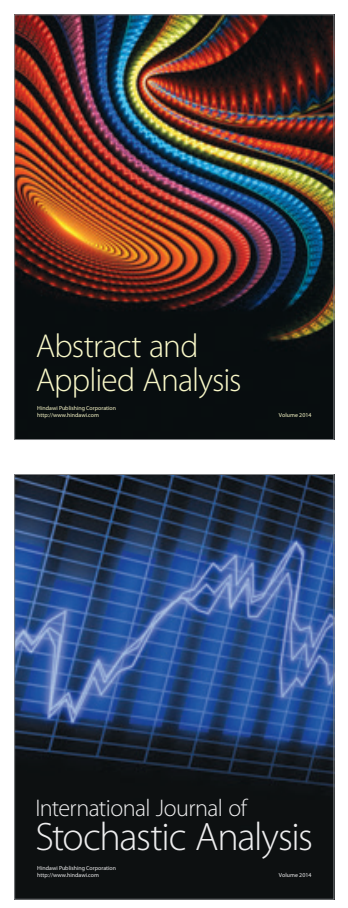

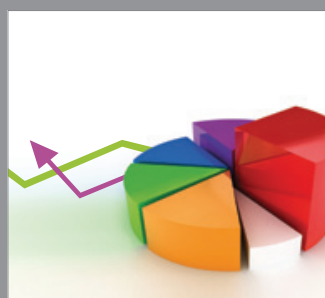

ournal of

Probability and Statistics

Promensencen
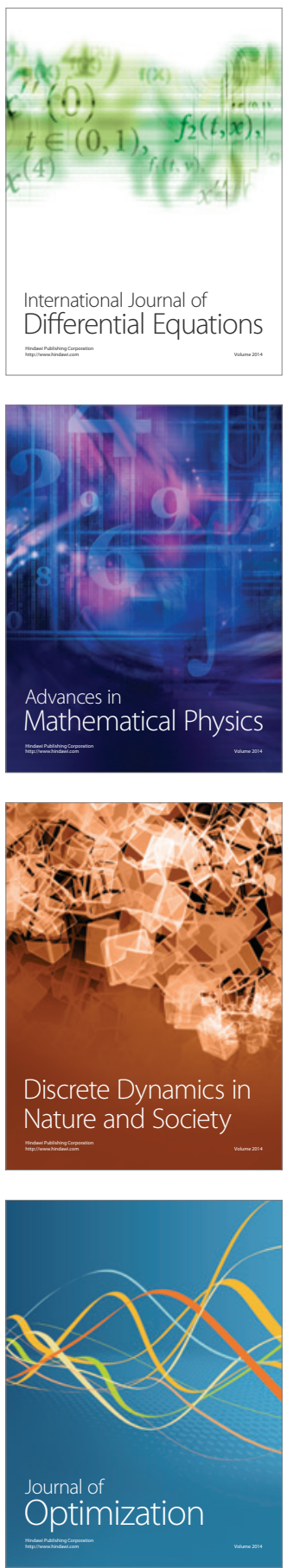